\title{
Sociotechnical Design of mHealth Applications for Chronic Diseases
}

\author{
Barbara Rita Barricelli \\ Dept of Computer Science \\ Università degli Studi di Milano \\ 20135 Milano, Italy \\ barricelli@di.unimi.it \\ Stefano Valtolina \\ Dept of Computer Science \\ Università degli Studi di Milano \\ 20135 Milano, Italy \\ valtolin@di.unimi.it \\ Jose Abdelnour-Nocera \\ School of Computing and \\ Engineering \\ University of West London \\ W5 5RF London, UK \\ M-ITI \\ Funchal, Portugal \\ jose.abdelnour-nocera@uwl.ac.uk
}

Permission to make digital or hard copies of part or all of this work for personal or classroom use is granted without fee provided that copies are not made or distributed for profit or commercial advantage and that copies bear this notice and the full citation on the first page. Copyrights for third-party components of this work must be honored. For all other uses, contact the Owner/Author.

uses, contact the Owner/Author.
Copyright is held by the owner/author(s).

MobileHCI '16 Adjunct, September 06-09, 2016, Florence, Italy ACM 978-1-4503-4413-5/16/09.

http://dx.doi.org/10.1145/2957265.2965009

\begin{abstract}
This workshop paper aims at briefly presenting the authors' previous experience in the field of sociotechnical design of mHealth applications and at illustrating the opportunity in joining forces of multidisciplinary researchers, domain experts, and practitioners for improving the field.
\end{abstract}

\section{Author Keywords}

Chronic Diseases; mHealth; Mobile Devices; Personal Health Records; Patient Engagement; Patient

Empowerment; Sociotechnical Design.

\section{ACM Classification Keywords}

H.5.m. Information interfaces and presentation (e.g., $\mathrm{HCI}$ ): Miscellaneous.

\section{mHealth for Chronic Diseases}

mHealth is aimed at providing remote health care by the use of mobile technology and devices. In [1] a systematic review on scientific literature about the management of chronic diseases has been presented and discussed. In particular, the review included the analysis of 107 papers and compared usability, feasibility, and acceptability of mobile tools and platform used. From the results, it emerged that the most commonly used mobile technology (40.2\%), was still Short Message Service (SMS). Particularly, about 
the practice of mHealth for anticoagulant therapy management (that is the specific application domain we consider in our past research), several studies have been carried out in the past years but none of them ended in actual implementation into practice (e.g., [2], $[3],[4])$.

The scientific literature available today strongly proves how the use of electronic management of health records can be of advantage both for patients and for healthcare practitioners, especially with what concerns a better management of National Health Systems (e.g., $[5],[6],[7])$.

By analyzing the existing literature and the outcomes of our previous research work we can state that there are two main advantages in implementing mHealth solutions for chronic diseases: the first is more related to its social impact and is the increasement of patients' awareness in their health condition. The second one is related to a reduction of costs for the National Health Systems. As a significant example, Javitt et al [8] shows that the adoption of mHealth technology for diabetes monitoring can lead to a significant reduction in medical expenditures, estimated in about $\$ 3,000$ per person per year.

At European level, the importance of mHealth and eHealth in general is largely recognized by the eHealth Action Plan 2012-2020 - Innovative healthcare for the 21st century [9]. In fact, in this period, until 2020, research and innovation will be supported under the programme "Health, demographic change and wellbeing" of Horizon 2020. Specifically, the research that will be funded has to be focused on (i) the exploitation of ICT and computer science for digital, personal, and predictive medicine, (ii) new tools and methods for improving diagnostics and decision making processes; (iii) the study of new mobile technologies and applications and of digital media in general to integrate existing healthcare and social care systems; (iv) the design of eHealth systems and services that enable a stronger user empowerment and involvement to move towards a more patient-centric healthcare system.

\section{The MANTRA Project Experience}

In 2013, two of the authors were involved in MANTRA (Mobile ANticoagulant TheRApy), a Leverlhume Trust funded project developed at University of West London, aimed at introducing mobile devices in anticoagulant therapy practice to improve the quality of life of patients. By the evaluation of the MANTRA Project, we developed a general approach to mHealth in the remote management of chronic diseases by supporting the communication among patients and healthcare practitioners. Patients usually have to make frequent visits to surgeries to meet nurses for the tests, and General Practitioners (GPs) for receiving drug prescriptions. The main goal of MANTRA was to study feasibility and acceptability of the introduction of mobile technology in the management of anticoagulant

therapy involving both patients and health practitioners in their design. The MANTRA Project design,

implementation and evaluation is reported as a proof of concept carried out in London [10] and also in

Venezuela, by exploring a specific case of mHealth in rural areas [11]. In particular, from the London-based case, the distinct characteristics of the NHS as one of the world's largest publicly funded health services, posed a number of sociotechnical challenges. We may classify into four main classes and generalize them 
from anticoagulant therapy to chronic diseases at large: 1) Flexibility: drugs prescription is subject to requirements the local national health system. 2) Accessibility: most of the patients who follow therapies for chronic diseases are elderly. 3) Data visualization: it is fundamental for the user to access history of therapy to learn from previous behavior and to monitor improvement or retrogression. 4) Safety: this is one of the most important characteristics that a mHealth application should have to be considered as appropriate for a clinical use.

The field of mHealth for chronic diseases management offers the opportunity to capitalize on a wide scientific literature production to improve the current medical practice, both in terms of doctor-patient communication and patient engagement/empowerment.

\section{Significance to the Workshop}

To participate to the workshop "Mobile Healthcare for the Self-Management of Chronic Diseases and the Empowerment of Patients" at MobileHCI 2016, will allow us to share with other scientists, domain experts, patients associations, and practitioners the challenges that we identified in our previous experience in the field. Moreover, this workshop will be an important occasion to eventually plan prospective future collaborations on the aspects of mHealth that strongly need further investigation:

- Method, techniques, technologies, and design approaches for improving the level of patients' engagement in therapy management in order to contribute to a higher efficacy and effectiveness of the cure and to a higher patients' satisfaction.
- Research or Industry case studies focused on improving patients' quality of life supporting tools for self-monitoring and self-management of their chronic diseases.

- Sociotechnical studies on methods for improving the quality of doctor-patient relationship enabling the construction of direct and fast communication channels focusing on data and avoiding time wasting.

\section{Acknowledgments}

The 2013 research work at the University of West London of Barbara Rita Barricelli on the MANTRA Project, supervised by Jose Abdelnour-Nocera, was supported by a Leverhulme Trust Visiting Fellowship.

\section{References}

1. Hamine, S., Gerth-Guyette, E., Faulx, D., Green, B. B., and Ginsburg, S. A. 2015. Impact of mhealth chronic disease management on treatment adherence and patient outcomes: A systematic review. J Med Internet Res. 17, 2, e52.

2. Gardiner, C., Williams, K., Mackie, I. J., Machin, S. J., and Cohen, H. 2006. Can oral anticoagulation be managed using telemedicine and patient selftesting? A pilot study. Clinical \& Laboratory Haematology 28, 2, 122-125.

3. Salvador, C. H., Ruiz-Sanchez, A., de Mingo, M. A. G., Carmona Rodriguez, M., Carrasco, M. P. Sagredo, P. G., Fragua, J. A., Caballero-Martinez, F., Garcia-Lopez, F., Marquez-Montes, J., and Monteagudo, J. L. 2008. Evaluation of a telemedicine-based service for the follow-up and monitoring of patients treated with oral anticoagulant therapy. Trans. Info. Tech. Biomed. $12,6,696-706$

4. Christensen, H., Lauterlein, J., Sørensen, P., Petersen, E., Madsen, J., and Brandslund, I. 2011. 
Home management of oral anticoagulation via telemedicine versus conventional hospitalbased

treatment. Telemedicine and eHealth 17, 3, 169176.

5. Cabitza, F., Simone, C., and De Michelis, G. 2015 User-driven prioritization of features for a prospective interpersonal health record. Comput. Biol. Med. 59, C, 202-210.

6. Chen, Y., Cheng, K., Tang, C., Siek, K. A., and Bardram, J. E. 2013. Is my doctor listening to me?: Impact of health it systems on patient-provider interaction. In $\mathrm{CHI}$ '13 Extended Abstracts on Human Factors in Computing Systems (CHI EA '13), 2419-2426.

7. Dedding, C., van Doorn, R., Winkler, L., and Reis, R. 2011. How will e-health affect patient participation in the clinic? a review of e-health studies and the current evidence for changes in the relationship between medical professionals and patients. Social Science \& Medicine 72, 49-53.

8. Javitt, J. C., Shane Reese, C., and Derrick, M. K. 2013. Deployment of an mhealth patient monitoring solution for diabetes improved glucose monitoring leads to reduction in medical expenditure. US Endocrinology 9, 2, 119-123.

9. Communication From The Commission To The European Parliament, The Council, The European Economic And Social Committee And The Committee Of The Regions Ehealth Action Plan 2012-2020 - Innovative Healthcare For The 21st Century. Online: http://eurlex.europa.eu/procedure/EN/202214

10. Barricelli, B. R., Devis, Y., Abdelnour-Nocera, J., Wilson, J., and Moore, J. 2013. Mantra: Mobile anticoagulant therapy management. In 2013 7th International Conference on Pervasive Computing Technologies for Healthcare and Workshops, 278281.
11. Barricelli, B.R., Devis, Y. 2014. mHealth in Resource-Constrained Environments. International Journal of Sociotechnology and Knowledge Development (IJSKD) 6, 1, 18-35. 